\title{
Transpulmonary pressures in obese and non-obese COVID-19 ARDS
}

\author{
Mehdi Mezidi 1,2, Florence Daviet ${ }^{3,4}$, Paul Chabert ${ }^{1}$, Sami Hraiech ${ }^{3,4}$, Laurent Bitker ${ }^{1,2,5}$, Jean-Marie Forel ${ }^{3,4}$, \\ Hodane Yonis' ${ }^{1}$, Ines Gragueb ${ }^{3}$, Francois Dhelft ${ }^{1}$, Laurent Papazian ${ }^{3,4}$, Jean-Christophe Richard ${ }^{1,2,5}$ \\ and Christophe Guervilly ${ }^{3,4^{*}}$ (1)
}

\begin{abstract}
Background: Data on respiratory mechanics of COVID-19 ARDS patients are scarce.Respiratory mechanics and response to positive expiratory pressure (PEEP) may bedifferent in obese and non-obese patients.

Methods: We investigated esophageal pressure allowing determination oftranspulmonary pressures $(\mathrm{PL})$ and elastances (EL) during a decremental PEEP trialfrom 20 to $6 \mathrm{~cm} \mathrm{H}_{2} \mathrm{O}$ in a cohort of COVID-19 ARDS patients.

Results: Fifteen patients were investigated, 8 obese and 7 non-obese patients. PEEP $\geq 16 \mathrm{~cm} \mathrm{H}_{2} \mathrm{O}$ for obese patients and PEEP $\geq 10 \mathrm{~cm} \mathrm{H}_{2} \mathrm{O}$ for non-obese patients were necessary to obtain positive expiratory $\mathrm{P}_{L}$. Change of PEEP did not alter significantly $\triangle P_{L}$ or elastances in obese patients. However, in non-obese patients lung $E L$ and $\triangle P_{L}$ increased significantly with PEEP increase. Chest wall EL was not affected by PEEP variations in both groups.
\end{abstract}

\section{Background}

Obesity, which is usually associated with better outcome for acute respiratory distress syndrome (ARDS) patients, is considered as a risk factor of acquiring a severe form of SARS COV-2-associated ARDS for SARS-CoV-2 [1]. Impact of obesity on respiratory mechanics of SARS$\mathrm{CoV}$-2-associated ARDS has not been investigated.

We hypothesized that respiratory mechanics including esophageal pressure (Pes) measurements might be different in obese and non-obese patients.

Therefore, the first objective of this study was to investigate transpulmonary pressures $\left(P_{L}\right)$ in intubated SARS$\mathrm{CoV}-2$ patients according to their body mass index (BMI) during a decremental PEEP trial. Secondary objective was to assess lung and chest wall elastances $\left(E L_{L}\right.$ and $\mathrm{EL}_{C W}$, respectively).

\footnotetext{
*Correspondence: christophe.guervilly@ap-hm.fr

${ }^{3}$ Assistance Publique - Hôpitaux de Marseille, Hôpital Nord, Médecine Intensive Réanimation, Chemin Des Bourrely, 13015 Marseille, France Full list of author information is available at the end of the article
}

\begin{abstract}
Methods
Patients

We conducted a prospective observational study in two intensive care units both in tertiary university hospitals (Hôpital de la Croix-Rousse, Hospices Civils de Lyon and Hôpital Nord, Assistance Publique-Hôpitaux de Marseille).

Patients were included in the study from 15 March to 15 April 2020 if they fulfilled inclusion criteria: adult admitted into the ICU for SARS-CoV-2, intubated and mechanically ventilated with moderate-to-severe ARDS criteria, sedated and paralyzed for clinical purpose and monitored by Pes catheter. As part of routine clinical management, we performed a decremental PEEP trial from 20 to $6 \mathrm{~cm} \mathrm{H}_{2} \mathrm{O}$ by $2 \mathrm{~cm} \mathrm{H}_{2} \mathrm{O}$-steps in each patient during volume-controlled ventilation while other parameters were kept constant.
\end{abstract}

\section{Esophageal pressure monitoring, transpulmonary pressures and elastances calculations}

Pes catheter (Nutrivent TM, Sidam, Mirandola, Italy, or C7680U (Marquat, Boissy-St-Leger, France) was in place. The correct placement of esophageal catheter was 
confirmed by presence of cardiac artifacts on the esophageal curve and by an occlusion test (expiratory hold on the ventilator) in passive conditions with gentle chest compression. The occlusion test was considered as positive when the correlation between $\Delta P_{e s}$ and $\Delta$ airway pressure (Paw) was 0.8-1.2. To avoid overestimation or underestimation of esophageal pressures, we inflated the esophageal balloon with the minimal filling volume among the recommended range for each catheter which was within the flat portion of the volume-pressure curve of the balloon $[2,3]$.

At each PEEP step, 2-s end-inspiratory occlusion pause allowed measurement of respiratory system (RS) and esophageal plateau pressure (Pplat and $P_{e s, \text { insp }}$, respectively), whereas 5-s end-expiratory occlusion pause allowed, respectively, measurement of RS and esophageal total PEEP (PEEP ${ }_{t o t}$ and $P_{\text {es,exp }}$ respectively). RS driving pressure $\left(\Delta P_{\mathrm{RS}}\right)$ was calculated as Pplat minus $\mathrm{PEEP}_{\text {tot. }}$ RS, chest wall and lung elastances $\left(\mathrm{EL}_{R S}, \mathrm{EL}_{C W}\right.$, and $\mathrm{EL}_{L}$, respectively) and elastance ratio were computed according standard formula.

$P_{L}$ absolute values were calculated as airway pressures minus esophageal pressures during inspiration $\left(P_{L, \text { insp }}=P\right.$ plat $\left.-P_{\text {es,insp }}\right)$ and expiration $\left(P_{L, \text { exp }}=\mathrm{PEEP}_{\text {tot }}{ }^{-}\right.$ $\left.P_{\text {es,exp }}\right)$, transpulmonary driving pressure $\left(\Delta P_{L}\right)=P_{L, \text { insp }}{ }^{-}$ $P_{L, \text { exp }}$. Elastance ratio derived $P_{L}\left(P_{L, E R}\right)$ was calculated as $P$ plat $\mathrm{x}\left(\mathrm{EL}_{L} / \mathrm{EL}_{R S}\right)$.

\section{Statistics}

Obesity was defined by a BMI $\geq 30$. Results are reported as medians [interquartile range] or count (percentage) and compared between groups by Mann-Whitney $\mathrm{U}$. Friedman test was used for repeated variables. $p$ value $<0.05$ was considered as significant.

\section{Results}

Fifteen patients were included in the study, 8 in the obese group (median BMI 34 [33-41]) and 7 in the non-obese group (mean BMI 26 [25-29]). Patient's characteristics were comparable between groups except for age (66 [53-73] years for non-obese group vs. 44 [39-49] years for obese group, $p=0.04$ ). Table 1 compares respiratory mechanics for each BMI group according to the PEEP levels. Figure 1a represents $P$ plat and $\Delta P_{\mathrm{RS}}$ for each BMI group according to the PEEP levels. Figure $1 \mathrm{~b}$ represents transpulmonary pressures and $\Delta P_{L}$.

$\mathrm{PEEP} \geq 16 \quad \mathrm{~cm} \quad \mathrm{H}_{2} \mathrm{O}$ for obese patients and $\mathrm{PEEP} \geq 10 \mathrm{~cm} \mathrm{H}_{2} \mathrm{O}$ for non-obese patients were necessary to obtain positive $P_{L \text {, exp }}$ (Fig. $1 \mathrm{~b}$ ). At $16 \mathrm{~cm} \mathrm{H}_{2} \mathrm{O}$ of PEEP, $71 \%$ of non-obese patients had $P_{L}$, insp $\geq 20 \mathrm{~cm} \mathrm{H}_{2} \mathrm{O}$ and $0 \%$ of obese patients, whereas with $P_{L, E R}$ was $\geq 20 \mathrm{~cm}$ $\mathrm{H}_{2} \mathrm{O}$ in, respectively, $86 \%$ of non-obese patients and $75 \%$ of obese patients. Change of PEEP did not alter significantly $\Delta P_{L}$ or elastances in obese patients (Table 1 ). However, in non-obese patients $\mathrm{EL}_{R S}$ and $\mathrm{EL}_{L}$ increased significantly with PEEP increase. $\mathrm{EL}_{C W}$ was not affected by PEEP variations in both groups.

Differences between obese and non-obese groups were significant at $18-20 \mathrm{~cm} \mathrm{H}_{2} \mathrm{O}$ of PEEP with higher Pplat, $\Delta P_{\mathrm{RS}}, P_{L, i n s p}, \Delta P_{L}$ in non-obese patients.

\section{Discussion}

During decremental PEEP trial, we found differences in transpulmonary pressures and respiratory mechanics in COVID-19 ARDS patients according to the presence of obesity. First, $P_{L, i n s p}, \Delta P_{L}$ were higher in non-obese patients at high PEEP $\left(\geq 18 \mathrm{~cm} \mathrm{H}_{2} \mathrm{O}\right)$, as $P$ plat and $\Delta P_{\mathrm{RS}}$. Second, $\mathrm{EL}_{C W}$ and $\mathrm{EL}_{L}$ were not statistically different between groups. However, increase of PEEP was significantly associated with an increase of $\mathrm{EL}_{L}$ in non-obese patients. Third, high PEEP levels (i.e., $16 \mathrm{~cm} \mathrm{H}_{2} \mathrm{O}$ ) were associated with potential injurious $P_{L, E R}\left(\geq 20 \mathrm{~cm} \mathrm{H}_{2} \mathrm{O}\right)$.

Preliminary studies with CT-scan have reported diffuse and bilateral pulmonary lesions during COVID-19 ARDS, which is usually associated with recruitability by PEEP in ARDS [4].

Recent studies using the same method to assess recruitability (recruitment-to-inflation ratio) reported conflicting data in those patients [5-7] with range from 17 to $56 \%$ of highly recruitable patients and possible less recruitable patients at a more advanced time point of the disease [5].

However, the lower driving pressures $\left(\Delta P_{R S}\right.$ and $\left(\Delta P_{L}\right)$ observed for obese patients cannot discriminate lung recruitment from less lung overdistension without appropriate evaluation (CT-scan or electrical impedance tomography for instance).

We did not check for airway flow limitation in all patients that could have led to overestimate lung and respiratory system elastances, in particular in obese patients.

Finally, we were not able to compare respiratory mechanics of this cohort with non COVID-19 ARDS patients.

In conclusion, assessment of respiratory mechanics of COVID-19 ARDS patients with transpulmonary pressure monitoring might be useful when targets of protective lung ventilation could not be reached. The characteristics of obesity on respiratory mechanics airway opening pressure, recruitability of COVID-19 ARDS patients need further investigations.

\section{Abbreviations}

ARDS: Acute respiratory distress syndrome; BMl: Body mass index; $\Delta P_{L}$ : Transpulmonary driving pressure; $\Delta P_{\mathrm{RS}}$ : Respiratory system driving pressure; $\mathrm{EL}_{C W}$ : Chest wall elastance; $\mathrm{EL}_{L}$ : Lung elastance; $\mathrm{EL}_{R S}$ : Respiratory system 
Table 1 Respiratory mechanics according to BMI group and PEEP level

\begin{tabular}{|c|c|c|c|c|c|c|c|c|c|}
\hline \multirow[t]{2}{*}{ VPariable } & \multirow[t]{2}{*}{ BMI group } & \multicolumn{8}{|l|}{ PEEP $\left(\mathrm{cm} \mathrm{H}_{\mathbf{2}} \mathrm{O}\right)$} \\
\hline & & 6 & 8 & 10 & 12 & 14 & 16 & 18 & 20 \\
\hline \multirow[t]{2}{*}{$\begin{array}{c}\text { Pplat (cm } \\
\left.\mathrm{H}_{2} \mathrm{O}\right)\end{array}$} & Non-obese & 15 [13.6-17.7] & $\begin{array}{l}16.3[16.2- \\
18.4]\end{array}$ & $\begin{array}{c}18[17.7- \\
21.1]\end{array}$ & $\begin{array}{c}21.8[21.1- \\
22.6]\end{array}$ & $\begin{array}{l}25.8[23.8- \\
25.9]\end{array}$ & $\begin{array}{l}29.9[28.6- \\
30.7]\end{array}$ & $\begin{array}{c}34[32.6- \\
35.4]\end{array}$ & $38[35.4-40.9]$ \\
\hline & Obese ${ }^{\$}$ & $\begin{array}{l}16.5[15.1- \\
17.3]\end{array}$ & $\begin{array}{c}17.5[16.5- \\
18.5]\end{array}$ & 20.5 [19-21] & $\begin{array}{l}22.5 \\
{[21-23.3]}\end{array}$ & $\begin{array}{c}24.5[23.8- \\
26.3]\end{array}$ & $\begin{array}{c}27[25.8- \\
28.5]\end{array}$ & $\begin{array}{l}29.5 \\
{[27-30.5]^{*}}\end{array}$ & $\begin{array}{l}31.5[28.8- \\
32.8]^{*}\end{array}$ \\
\hline \multirow[t]{2}{*}{$\begin{array}{l}\text { Total PEEP } \\
\qquad\left(\mathrm{cm} \mathrm{H}_{2} \mathrm{O}\right)\end{array}$} & Non-obese & $6.8[6.8-6.9]$ & $9[8.4-9.5]$ & $\begin{array}{l}10.9 \\
{[10.9-11]}\end{array}$ & $\begin{array}{l}12.2[12.2- \\
13.3]\end{array}$ & 15 [15-15] & $\begin{array}{l}16.3[16.3- \\
16.7]\end{array}$ & 19 [19-19] & $\begin{array}{l}20.4[20.4- \\
21.4]\end{array}$ \\
\hline & Obese ${ }^{\xi}$ & $6.9[6.7-6.9]$ & 8.7 [8.3-8.9] & 10.5 [10-11] & 13 [12.2-13] & 15 [14-15] & $16.7[16-17]$ & 18.5 [18-19] & 20 [20-20.6] \\
\hline \multirow[t]{2}{*}{$\Delta P_{\mathrm{RS}}\left(\mathrm{cm} \mathrm{H}_{2} \mathrm{O}\right)$} & Non-obese & $8.2[6.8-10.2]$ & 6.8 [6.8-8.9] & 7 [6.8-9.6] & $8.2[8.2-10.5]$ & $\begin{array}{l}10.8 \\
{[8.8-11.4]}\end{array}$ & $\begin{array}{l}13.6[11.6- \\
14.5]\end{array}$ & $\begin{array}{c}15[13.6- \\
16.9]\end{array}$ & $18[14.3-20.2]$ \\
\hline & Obese & 9.6 [7.6-10.5] & 8.15 [7.7-9.9] & 10 [8-11] & 9 [8-12] & 10 [8.8-12.3] & 11 [8.8-12.3] & $\begin{array}{l}10.5 \\
{[8.1-12.5]^{*}}\end{array}$ & $11.5[8-13.3]^{*}$ \\
\hline \multirow[t]{2}{*}{$\begin{array}{l}P_{\text {es. insp }}(\mathrm{cm} \\
\left.\mathrm{H}_{2} \mathrm{O}\right)\end{array}$} & Non-obese $^{\xi}$ & 8.6 [6.9-10.2] & $9.4[7.6-10.9]$ & $10[7.6-11.6]$ & $\begin{array}{l}10.9 \\
{[8.8-11.6]}\end{array}$ & $\begin{array}{l}10.9 \\
{[8.8-12.8]}\end{array}$ & $\begin{array}{l}10.9 \\
{[9.3-12.8]}\end{array}$ & 12 [9.5-12.9] & 13 [10.9-14.3] \\
\hline & Obese & $\begin{array}{l}11.5 \\
{[9.1-14.5]}\end{array}$ & $\begin{array}{l}11.5 \\
{[9.5-15.3]}\end{array}$ & $\begin{array}{l}11.5 \\
{[9.5-16.3]}\end{array}$ & 12 [9.5-16] & $\begin{array}{l}12.5[10.5- \\
16.3]\end{array}$ & $\begin{array}{l}12.5[10.6- \\
16.5]\end{array}$ & $\begin{array}{c}13[11.4- \\
17.3]\end{array}$ & $\begin{array}{l}13.5[12.8- \\
17.5]\end{array}$ \\
\hline \multirow{2}{*}{$\begin{array}{l}P_{\operatorname{es} \cdot \exp }(\mathrm{cm} \\
\left.\mathrm{H}_{2} \mathrm{O}\right)\end{array}$} & Non-obese & $7.1[5.2-8.9]$ & 7.5 [5.9-8.9] & 7.8 [6.4-10.2] & $8.3[7.1-10.2]$ & 9.1 [7.1-10.9] & $9.5[7.6-11]$ & $10[8.1-11.6]$ & 11 [8.8-12.2] \\
\hline & Obese $e^{\xi}$ & $9.9[8.1-12.3]$ & $10.5[8.2-13]$ & $\begin{array}{l}10.5 \\
{[8.5-13.3]}\end{array}$ & 11 [8.7-14] & 11 [8.7-15] & $\begin{array}{l}11.5 \\
{[9.3-14.5]}\end{array}$ & $\begin{array}{l}11.5[10.3- \\
15.3]\end{array}$ & $12.5[11-16]$ \\
\hline \multirow[t]{2}{*}{$\begin{array}{c}P_{\text {Linsp }}(\mathrm{cm} \\
\left.\mathrm{H}_{2} \mathrm{O}\right)\end{array}$} & Non-obese & $6.8[6.4-9.3]$ & 8.2 [6.8-10.3] & $9.6[9.5-11.5]$ & $\begin{array}{l}10.9 \\
{[10.9-15]}\end{array}$ & $\begin{array}{l}14.9[12.9- \\
16.4]\end{array}$ & $\begin{array}{l}20.4[17.7- \\
20.7]\end{array}$ & $\begin{array}{l}24.5 \\
{[21.1-24.8]}\end{array}$ & $28[23.1-28.3]$ \\
\hline & Obese & $5.5[2.8-9]$ & $7[4.3-9.3]$ & $9[6.5-11.3]$ & $10[8.5-12.4]$ & $12.6[9.5-15]$ & $\begin{array}{l}14.8 \\
{[12.3-17]}\end{array}$ & $\begin{array}{l}16.2 \\
{[13-18.5]^{*}}\end{array}$ & $\begin{array}{l}17.15 \\
{[14.8-19]^{*}}\end{array}$ \\
\hline \multirow[t]{2}{*}{$P_{L, E R}\left(\mathrm{~cm} \mathrm{H}_{2} \mathrm{O}\right)$} & Non-obese & $\begin{array}{l}10.8 \\
{[9.4-15.4]}\end{array}$ & $\begin{array}{l}11.9[10.6- \\
14.3]\end{array}$ & $\begin{array}{l}15.4[14.1- \\
17.3]\end{array}$ & $\begin{array}{c}17.6[15.9- \\
19.2]\end{array}$ & $\begin{array}{c}20.5[18.5- \\
22.1]\end{array}$ & $\begin{array}{l}25.7[23.3- \\
27.7]\end{array}$ & $\begin{array}{l}31.1 \\
{[26-32.4]}\end{array}$ & $\begin{array}{l}33.8[28.6- \\
37.4]\end{array}$ \\
\hline & Obese & 13 [11.8-14.2] & $\begin{array}{l}13.8[12.8- \\
15.7]\end{array}$ & $\begin{array}{c}17.2[14.5- \\
19.1]\end{array}$ & $\begin{array}{l}18.4[15.7- \\
20.9]\end{array}$ & $\begin{array}{l}21.7[18.3- \\
22.8]\end{array}$ & $\begin{array}{c}24[20.3- \\
25.9]\end{array}$ & $\begin{array}{l}25.3[21.3- \\
27.4]\end{array}$ & $\begin{array}{l}27.8[23.8- \\
29.4]\end{array}$ \\
\hline \multirow[t]{2}{*}{$\begin{array}{c}P_{\text {L.exp }}(\mathrm{cm} \\
\left.\mathrm{H}_{2} \mathrm{O}\right)\end{array}$} & Non-obese & $\begin{array}{l}0[-1.9 \text { to } \\
1.7]\end{array}$ & $\begin{array}{l}1.4[-0.2 \text { to } \\
3.4]\end{array}$ & $3.2[1.4-4.6]$ & $4.1[2.7-5.4]$ & $5.9[4.2-7.4]$ & $7.3[5.5-8.6]$ & $9[7.5-10.4]$ & $10[8.9-11.5]$ \\
\hline & Obese ${ }^{\xi}$ & $\begin{array}{c}-3.1[-5.4 \\
\text { to }-1.3]\end{array}$ & $\begin{array}{l}-1.9[-4.2 \\
\text { to } 0.5]\end{array}$ & $0[-3$ to 1.8$]$ & $1[-1$ to 3.8$]$ & $\begin{array}{l}3.9[-0.3 \text { to } \\
5.8]\end{array}$ & $5[1.8-7]$ & $7[3.5-7.6]$ & $7[4.8-9]^{*}$ \\
\hline \multirow[t]{2}{*}{$\Delta P_{L}$} & Non-obese & $5.5[4.8-8.9]$ & $5.4[4.6-6.8]$ & $6[5.4-7.8]$ & 6.9 [6.2-8.8] & $9.1[6.8-9.4]$ & 12 [9.5-13] & $\begin{array}{l}13.7[10.9- \\
15.5]\end{array}$ & $16[11.5-18.5]$ \\
\hline & Obese & $7.6[5.9-8.7]$ & $6.7[5.9-8.2]$ & $8[6.5-10]$ & $7.5[5.8-10.3]$ & $9[6.8-10.4]$ & 9.4 [7.5-11.3] & 9 [6.6-11.3] & $10.5[6.8-11.3]^{*}$ \\
\hline \multirow[t]{2}{*}{$\begin{array}{l}\mathrm{EL}_{R S}\left(\mathrm{~cm} \mathrm{H}_{2} \mathrm{O} .\right. \\
\left.\mathrm{L}^{-1}\right)\end{array}$} & Non-obese & $20[17.4-23.8]$ & $\begin{array}{l}18.3[15.6- \\
23.2]\end{array}$ & $\begin{array}{l}18.3 \\
{[17-23.3]}\end{array}$ & $\begin{array}{l}24.1[18.4- \\
26.4]\end{array}$ & $\begin{array}{c}24[22.7- \\
28.9]\end{array}$ & $\begin{array}{c}33.3[27.4- \\
38.3]\end{array}$ & 39.3 [32-43] & $\begin{array}{l}47.1[33.5- \\
54.1]\end{array}$ \\
\hline & Obese & $\begin{array}{c}27.3[18.5- \\
38.1]\end{array}$ & $\begin{array}{l}26.3 \\
{[18-38.3]}\end{array}$ & $\begin{array}{l}25.1[17.6- \\
53.7]\end{array}$ & $\begin{array}{l}26.7 \\
{[18-49.1]}\end{array}$ & $\begin{array}{c}30.1[18.6- \\
48.5]\end{array}$ & $\begin{array}{l}31.4[19.9- \\
53.4]\end{array}$ & $\begin{array}{l}29.2[23.3- \\
45.9]\end{array}$ & $\begin{array}{l}31.4[22.9- \\
45.9]\end{array}$ \\
\hline \multirow{2}{*}{$\begin{array}{l}\mathrm{EL}_{\mathrm{CW}}(\mathrm{cm} \\
\left.\mathrm{H}_{2} \mathrm{O} \cdot \mathrm{L}^{-1}\right)\end{array}$} & Non-obese & $4.6[3.8-5.7]$ & $5.8[4.7-6.2]$ & $3.2[3.1-5.2]$ & $5.2[3.5-6.3]$ & $5.2[3.6-6.3]$ & $5.2[3.6-6.3]$ & $3.8[3.2-6.2]$ & $6.2[4.7-6.3]$ \\
\hline & Obese & $4.2[4-5.7]$ & $4.2[3.5-7.1]$ & $4.8[2.7-7.8]$ & $4.9[2.7-8.2]$ & $4.8[4.2-8.4]$ & $5.7[2.7-8.2]$ & $4.2[3.5-6.7]$ & 3.9 [2.7-6.7] \\
\hline \multirow[t]{2}{*}{$\begin{array}{c}\mathrm{EL}_{L}\left(\mathrm{~cm} \mathrm{H}_{2} \mathrm{O} .\right. \\
\left.L^{-1}\right)\end{array}$} & Non-obese & $\begin{array}{l}15.9[11.6- \\
20.7]\end{array}$ & $\begin{array}{l}13.1[10.9- \\
17.8]\end{array}$ & $\begin{array}{l}15.7[13.7- \\
19.6]\end{array}$ & $\begin{array}{l}20.3[13.8- \\
22.2]\end{array}$ & $\begin{array}{l}20.9[17.7- \\
24.6]\end{array}$ & $\begin{array}{c}30.2[22.5- \\
33.8]\end{array}$ & $\begin{array}{c}36.4[25.7- \\
39.8]\end{array}$ & $\begin{array}{c}41.9[27.2- \\
48.7]\end{array}$ \\
\hline & Obese & $\begin{array}{c}23.3[14.6- \\
32.4]\end{array}$ & $\begin{array}{c}22.3[15.3- \\
30.7]\end{array}$ & $\begin{array}{l}22.6 \\
{[14.9-42]}\end{array}$ & $\begin{array}{c}23.2[12.8- \\
39.8]\end{array}$ & $\begin{array}{c}26.4[14.2- \\
42.5]\end{array}$ & $\begin{array}{c}28.9[16.1- \\
43.6]\end{array}$ & $\begin{array}{c}25.2[19.9- \\
36.6]\end{array}$ & $\begin{array}{c}28.9[20.3- \\
38.8]\end{array}$ \\
\hline \multirow[t]{2}{*}{ EL ratio } & Non-obese & $\begin{array}{c}0.79[0.67- \\
0.87]\end{array}$ & $\begin{array}{c}0.75[0.66- \\
0.80]\end{array}$ & $\begin{array}{c}0.8[0.79- \\
0.84]\end{array}$ & $\begin{array}{c}0.81[0.74- \\
0.84]\end{array}$ & $\begin{array}{c}0.83[0.74- \\
0.85]\end{array}$ & $0.86[0.8-0.9]$ & $\begin{array}{c}0.9[0.79- \\
0.92]\end{array}$ & $0.89[0.81-0.9]$ \\
\hline & Obese & $\begin{array}{c}0.8[0.77- \\
0.85]\end{array}$ & $\begin{array}{c}0.80[0.78- \\
0.85]\end{array}$ & $\begin{array}{c}0.84[0.74- \\
0.89]\end{array}$ & $\begin{array}{c}0.82[0.72- \\
0.89]\end{array}$ & $\begin{array}{c}0.85 \text { [0.77- } \\
0.86]\end{array}$ & $\begin{array}{l}0.87 \\
{[0.79-0.9]}\end{array}$ & $0.85[0.8-0.9]$ & $\begin{array}{c}0.88[0.79- \\
0.91]\end{array}$ \\
\hline
\end{tabular}

Variables are reported as medians [interquartile range]

$B M I$ body mass index, $P E E P$ positive end-expiratory pressure, $P$ plat plateau pressure, $\Delta P_{R S}$ respiratory system driving pressure, $P_{\text {es. insp }}$ esophageal inspiratory pressure, $P_{\text {es exp }}$ esophageal expiratory pressure, $P_{L \text {. insp }}$ inspiratory transpulmonary pressure, $P_{L, E R}$ transpulmonary pressure calculated with the elastance ratio, EL ratio, $P_{L \text {.exp }}$ expiratory transpulmonary pressure, $\Delta P_{L}$ driving transpulmonary pressure, $E L_{R S}$ respiratory system elastance, $E L_{C W} c h e s t$ wall elastance, $E L_{L}$ lung elastance, $E L$ ratio, ratio of lung elastance to elastance of the respiratory system.

${ }^{*} p<0.05$ between obese and non-obese group for a given PEEP by Mann-Whitney $U$ test; ${ }^{\$} p<0.05$ for PEEP effect by Friedman test. Computed for each group 

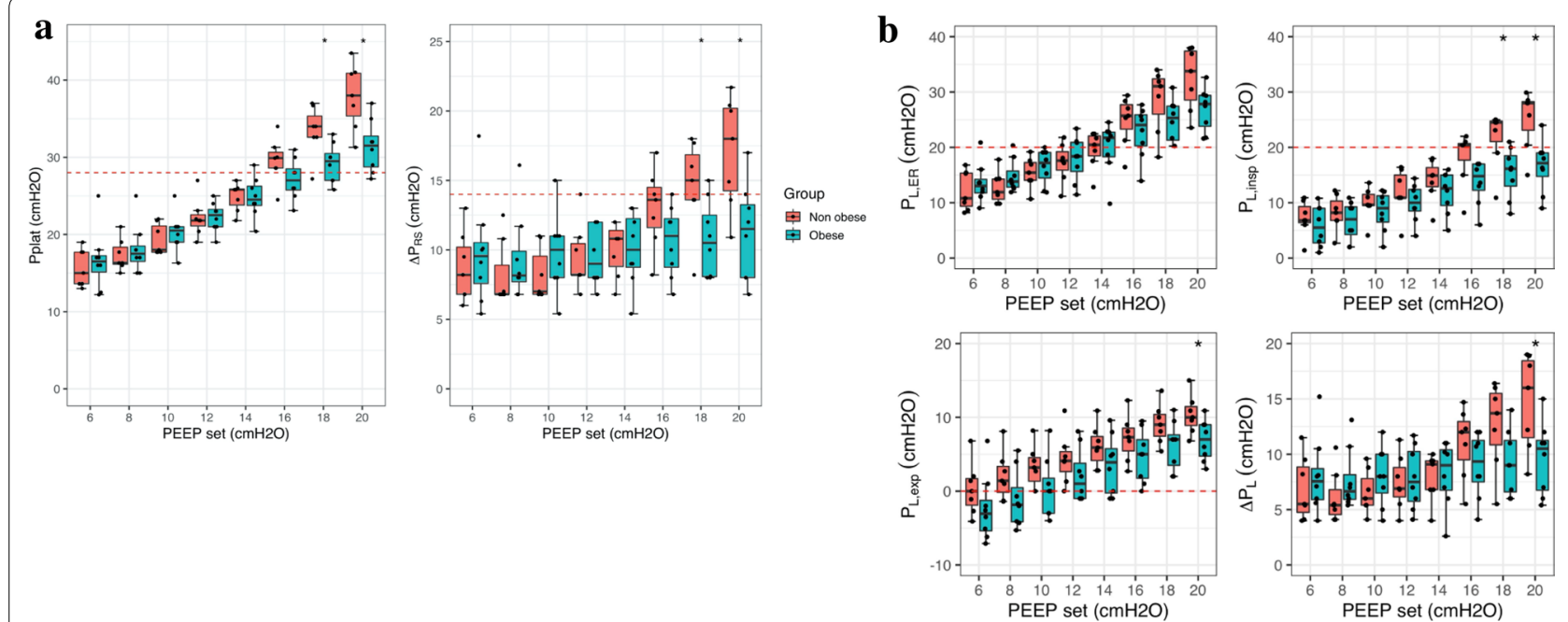

Group 审 Non obese 审 Obese

Fig. 1 a Boxplots of respiratory system plateau pressures (Pplat) according to set PEEP and group. Black dots represent individual values. Red dashed line denotes $28 \mathrm{~cm} \mathrm{H}_{2} \mathrm{O}$. ${ }^{*} p<0.05$ between obese and non-obese group for a given PEEP by Mann-Whitney $U$ test. Boxplots of respiratory system driving pressures $\left(\triangle \mathrm{P}_{\mathrm{RS}}\right)$ according to set PEEP and group. Black dots represent individual values. Red dashed line denotes $14 \mathrm{~cm} \mathrm{H}_{2} \mathrm{O}$. ${ }^{*} p<0.05$ between obese and non-obese group for a given PEEP by Mann-Whitney $U$ test. $\mathbf{b}$ Boxplots of end-inspiratory elastance-ratio derived $\left(P_{L, E R}\right)$, end-inspiratory absolute method $\left(P_{L, \text { insp }}\right)$ and end-expiratory $\left(P_{L, \text { exp }}\right)$ transpulmonary pressures according to set PEEP and group. Black dots

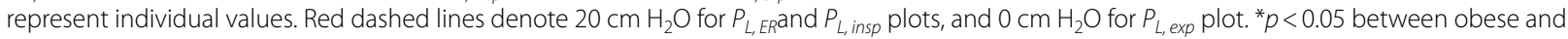
non-obese group for a given PEEP by Mann-Whitney $U$ test

elastance; ICU: Intensive care unit; PEEP: Positive end-expiratory pressure; Pes: Esophageal pressure; $P_{L, E R}$ : Inspiratory transpulmonary pressure (elastance ratio); $P_{L, \text { exp }}$ : Expiratory transpulmonary pressure; $P_{L, \text { insp: }}:$ Inspiratory transpulmonary pressure (absolute value); $P_{L}:$ Transpulmonary pressure; Pplat: Plateau pressure; SARS-CoV-2: Severe acute respiratory syndrome coronavirus.

\section{Acknowledgements}

The authors want to acknowledge Sabine Valera for the English editing and all the nurses, caregivers, physiotherapists, doctors and support peoples involved in the COVID-19 crisis in both centers.

\section{Authors' contributions}

MM and CG were responsible for the concept, revision and approval of this manuscript. MM, SH and CG participated in the design and drafted the manuscript. LP and JCR helped to revise the manuscript. MM, FD, PC, SH, LB, JMF, HY, IG, FD, LP, JCR and CG contributed to the data analysis and interpretation. All authors read and approved the final manuscript.

\section{Funding}

No specific funding was used for this study.

\section{Availability of data and materials}

The datasets analyzed during the current study are available from the corresponding author on reasonable request.

\section{Ethics approval and consent to participate}

All patients admitted for SARS-CoV-2 or their relatives in case of unable to be informed patients, received non-opposition information to be part of the French clinical data base (COVID-ICU). The study was approved by an Ethic Committee, through the French national registry COVID-ICU for patients included in Marseille and through the Lyon University for patients included in Lyon (\#20-41).

\section{Consent for publication}

All authors reviewed the manuscript and approved the publication of the manuscript. The corresponding author has completed the "Consent for publication".

\section{Competing interests}

The authors have no competing interests to declare.

\section{Author details}

${ }^{1}$ Medical ICU, Croix-Rousse Hospital, Hospices Civils de Lyon, Lyon, France. ${ }^{2}$ Lyon 1 University, Lyon, France. ${ }^{3}$ Assistance Publique - Hôpitaux de Marseille, Hôpital Nord, Médecine Intensive Réanimation, Chemin Des Bourrely, 13015 Marseille, France. ${ }^{4}$ Aix-Marseille Université, Faculté de médecine, Centre d'Etudes et de Recherches sur les Services de Santé et qualité de vie EA 3279 . 13005 Marseille, France. ${ }^{5}$ CREATIS, CNRS UMR5220, INSERM U1044, INSA, Lyon, France.

Received: 22 April 2020 Accepted: 23 September 2020

Published online: 01 October 2020

\section{References}

1. Simonnet A, Chetboun M, Poissy J, et al. High prevalence of obesity in severe acute respiratory syndrome coronavirus-2 (SARS-CoV-2) requiring invasive mechanical ventilation. Obesity. 2020. https://doi.org/10.1002/ oby.22831.

2. Akoumianaki E, Maggiore SM, Valenza F, et al. The application of esophageal pressure measurement in patients with respiratory failure. Am J Respir Crit Care Med. 2014 Mar 1;189(5):520-31. https://doi.org/10.1164/ rccm.201312-2193Cl.ReviewPubMed PMID: 24467647. 
3. Mojoli F, lotti GA, Torriglia F, et al. In vivo calibration of esophageal pressure in the mechanically ventilated patient makes measurements reliable. Crit Care. 2016;20:98. https://doi.org/10.1186/s13054-016-1278-5.

4. Chiumello D, Marino A, Brioni M, et al. Visual anatomical lung CT scan assessment of lung recruitability. Intensive Care Med. 2013;39(1):66-73. https://doi.org/10.1007/s00134-012-2707-9.

5. Pan C, Chen L, Lu C, et al. Recruitability in SARS-CoV-2 associated acute respiratory distress syndrome: a single-center, observational study. Am J Respir Crit Care Med. 2020. https://doi.org/10.1164/rccm.202003-0527L E[Epub ahead of print] PubMed PMID: 32200645

6. Beloncle FM, Pavlovsky B, Desprez C, et al. Recruitability and effect of PEEP in SARS-Cov-2-associated acute respiratory distress syndrome. Ann
Intensive Care. 2020;10(1):55 https://doi.org/10.1186/s13613-020-00675

7. Haudebourg AF, Perier F, Tuffet S, et al. Respiratory Mechanics of COVID19 vs. Non-COVID-19 associated acute respiratory distress syndrome. Am J Respir Crit Care Med. 2020. https://doi.org/10.1164/rccm.202004-1226L E.

\section{Publisher's Note}

Springer Nature remains neutral with regard to jurisdictional claims in published maps and institutional affiliations.

\section{Submit your manuscript to a SpringerOpen ${ }^{\circ}$ journal and benefit from:}

- Convenient online submission

- Rigorous peer review

- Open access: articles freely available online

- High visibility within the field

- Retaining the copyright to your article

Submit your next manuscript at $\boldsymbol{\nabla}$ springeropen.com 